\title{
Searching the evidence: the clinically-effective nutritionist
}

\author{
Helen Moore and Carolyn Summerbell* \\ Postgraduate Institute, School of Health and Social Care, University of Teesside, Middlesbrough TS1 3BA, UK
}

\begin{abstract}
As a clinically-effective nutritionist faced with needing to find the answer to a clinical question quickly it is necessary to search the best evidence efficiently. The requirements are to: (1) avoid having lots of papers to read; (2) be able to access this information at one's place of work; (3) restrict reading to trials and systematic reviews of trials. If a decision has to be made while on the ward, the best resource is probably an easy-to-use book, Clinical Evidence. This resource is also available on-line, but there may not be access to the Internet on the ward. If a little more time is available, and access to the Internet, the following plan is suggested: (1) work out what the question is and highlight the search terms; (2) using the best search engine available, search MEDLINE from 1990 for titles and abstracts of papers containing the search terms, this is the search strategy (limit the search by publication type by requesting randomised controlled trials only, English language only and human only); (3) if more or less than approximately ten hits are obtained, alter the limits of the search (not the search strategy); (4) read the abstract, or full paper where available, of the relevant hits and appraise this evidence.
\end{abstract}

\section{Search strategy: Clinical effectiveness: Evidence-based practice}

\section{What is evidence?}

Any nutritional intervention has benefits and harmful effects that can be evaluated through research. This research is nearly always published in some form: internal reports, abstracts at conferences, published papers. Over two million biomedical papers are published each year, making it impossible for nutritionists to keep up to date with current knowledge. The clinically-effective nutritionist needs to be able to search for and assess the best evidence on any particular problem, quickly. Best evidence:

depends on the type of question being asked; e.g. a common type of question asked by a clinical nutritionist is: how effective is a particular nutritional intervention?;

for questions about the effectiveness of a nutritional intervention are systematic reviews of randomised controlled trials, then individual randomised controlled trials.

for questions about the effectiveness of a nutritional intervention where randomised controlled trials are not available or not appropriate (e.g. community nutrition interventions), 'controlled' studies should be considered (with caution because of increased risk of bias).

\section{Smart searching for best evidence}

In the present paper the aim is to provide some tips on how to be smart about searching for best evidence. Two routes for this process will be suggested. The first is based on the premise that $30 \mathrm{~min}$ are available to find the answer. The second is based on the premise that only $10 \mathrm{~min}$ are available. Both routes rely on the fact that there is Internet access, but otherwise these resources are free.

\section{Smart searching for best evidence in $\mathbf{3 0} \mathrm{min}$}

Searching the health care literature is an activity in which health care professionals must routinely engage to seek evidence-based answers to specific practice questions and also to stay abreast of developments in the field. The present paper will illustrate techniques for searching the Cochrane Library and MEDLINE (via PubMed) efficiently, as a means of answering a specific clinical question. There are, of course, many other useful bibliographic databases. However, access to both the Cochrane Library and MEDLINE (via PubMed) are free to anyone in the UK and once the basic concepts of searching these two databases have been mastered, those skills will quickly adapt to any other database that may be found and considered worth searching. The 


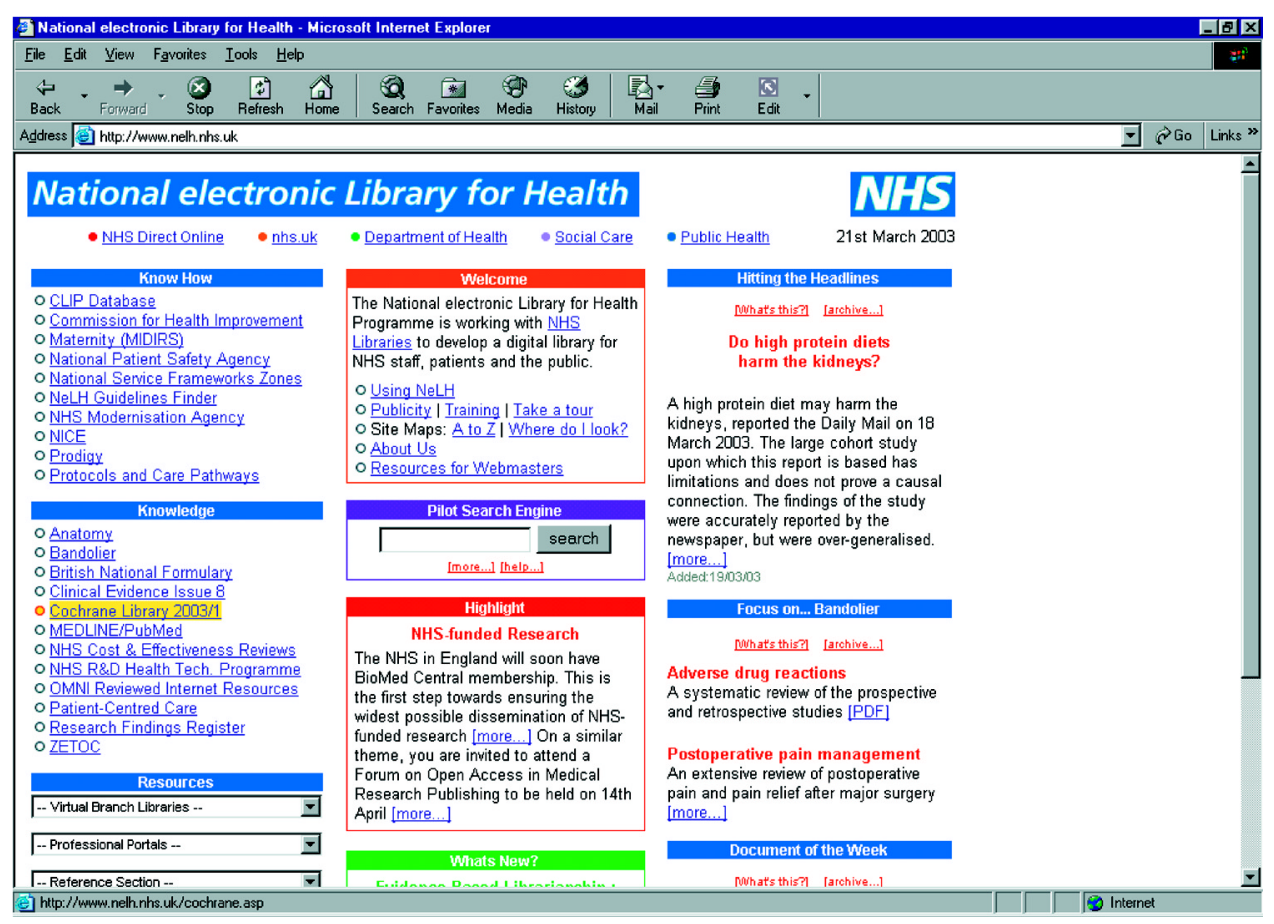

Fig. 1. Home page for the National electronic Library for Health.

gateway to both these databases (and many other useful databases) is the National electronic Library for Health: http://www.nelh.nhs.uk (see Fig. 1).

The order in which the databases are searched is important. If a systematic review of the intervention being looked at has already been carried out, there is little point in re-inventing the wheel and looking for and evaluating all the empirical data. Thus, begin by looking at whether there is a systematic review on the subject and by searching the Cochrane Library.

\section{The Cochrane Library}

The Cochrane Library is compiled by the Cochrane Collaboration and the National Health Service Centre for Reviews and Dissemination. It contains within it four databases:

1. the Cochrane Database of Systematic Reviews, which contains the full text of all completed reviews carried out by the Cochrane Collaboration and also full text of all protocols for reviews that are currently in preparation;

2. the Database of Abstracts of Reviews of Effectiveness, which contains abstracts of other systematic reviews (i.e. non-Cochrane reviews);

3. the National Health Service Economic Evaluation Database, which is a register of published economic evaluations of health care interventions;

4. the Cochrane Controlled Trials Register, which is a database that contains references to randomised controlled trials that have been identified through hand-searching of journals and other databases.
The aim of the Cochrane Library is to make results of high-quality systematic reviews and randomised controlled trials readily available. It aims to help to get good research into practice. It is particularly useful when searching for information on the effectiveness of a particular nutritional intervention.

\section{Searching the Cochrane Library}

The search engine within the Cochrane Library is relatively crude (compared with MEDLINE). Within the search box at the top left hand corner of the screen, type in a keyword. To limit the search, click on the arrow next to the words 'refine your search'. A drop-down menu will appear that will allow the search to be limited by the particular years over which the search is to be made. Then, by clicking 'go' the number of hits within each of the databases will be identified. To look at these hits, click on each database in turn. To look at each hit in detail, click on the hit that is to be viewed.

\section{MEDLINE}

MEDLINE is compiled by the US National Library of Medicine. It is the electronic version of Index Medicus and Index to Dental Literature. It indexes over 3900 journals and comprises articles published in these journals from 1966 to the present day.

Different interfaces are available to access MEDLINE. PubMed is an interface that is available free of charge. The OVID interface, for example, is better than PubMed (the logic with which the search is processed is better), but it is not free. 


\section{Search strategy for MEDLINE}

To create a search strategy the following plan is suggested:

1. compose the question;

2. analyse the elements of the question in the format based on patient or condition, intervention, comparison, outcome (PICO), which is an easy guide to the first step of turning your clinical question into an effective search strategy;

3. select search terms from this question for use in the search strategy.

To illustrate this process, the following clinical scenario will be used: A 60-year-old man with type 2 diabetes attends his regular appointment at an outpatient diabetes clinic. Recently, he stopped smoking and was successful. He has not smoked for 6 months, but has gained $9.5 \mathrm{~kg}$ in weight, and his BMI is now $32 \mathrm{~kg} / \mathrm{m}^{2}$. His diet is not too bad, but could certainly be improved. He is not particularly active, although he does play golf once weekly. He makes most walkable journeys by car.

The first line of treatment for this man should address dietary intervention. Thus, the clinical question in PICO format is:

$\mathrm{P}$ : in a 60-year-old overweight man with type 2 diabetes who has recently given up smoking (overweight; type 2 diabetes);

I: what type of dietary advice (diet);

$\mathrm{C}$ : when compared with no intervention;

$\mathrm{O}$ : produces the greatest weight loss and an improvement

in glycaemic control (weight loss; glycaemic control).

These search terms may be known by other names. It is important to search using all possible synonyms. The search terms should therefore include other names for overweight (obese, obesity), type 2 diabetes (NIDDM, non-insulindependent diabetes (with or without the hyphens) and type II diabetes), glycaemic (glycemic). As with the last example, it is worth considering the US spelling of the term. In addition, the use of 'wild cards' can be helpful. For example, a search on diet* will return all hits with words prefixed with diet (e.g. diet, dietary, dietitian). Another useful tool is the use of inverted commas around a string of words. For example, a search on "weight loss" will only return hits where the words weight and loss are next to each other as weight loss.

\section{How to search MEDLINE}

There are a number of different methods that can be used to search MEDLINE; use of free text words, keywords or medical subject headings. It is possible to combine any of these types of searching. It is recommended that free text searching is used.

Free text searching. Searching using free text words means that the search terms are typed in, the database trawls the titles and abstracts of the indexed papers and wherever the text word is mentioned that article is returned by the database. This procedure is a very useful way to search MEDLINE. However, the database only returns articles with the exact words used, and therefore relies on the author using exactly the same word(s) as that identified as the search term.

Keyword searching. Keyword searching relies on the author's use of words in the title and/or abstract.

Subject searching. This method is a very powerful way of searching the database. It is based on an index of medical subject headings. When searching using this method, articles are retrieved based on the keywords that were assigned to them. These keywords are assigned based on the article's content, not according to the words that were used by the author in the article. It is possible to broaden or narrow the search by 'exploding' or 'focusing' the search.

Boolean operators. Regardless of which MEDLINE interface is used, it will be necessary to be able to combine various concepts using Boolean operators. The most common of these operators are 'AND', 'OR' and 'NOT'. 'AND' and 'OR' are both combining operators, but they do have slightly different modes of operation. 'OR' looks for articles that contain search term 1 OR search term 2, whereas if the terms are combined with the 'AND' operator, only those articles that have both terms in them are returned. The 'NOT' operator is an exclusion operator, e.g. this operator would be used if the requirement is to view articles that contain the first search term and not the second.

Limits. In MEDLINE it is possible to limit the search in a number of ways. For the example described earlier the search has been limited to (1) field: abstract and title; (2) publication date: from 1996 to present; (3) only items with abstracts; (4) English language only; (5) publication type: randomised controlled trials only; (6) human only.

\section{Searching MEDLINE (through PubMed)}

First, click on 'Limits', and limit the search. Then enter all the search terms one by one into the search box at the top of the screen. Then click the 'History' link so that the process of combining the searches can take place. For the example given earlier, the following hits were returned:

$\begin{array}{lr}\text { 1. overweight } & 239 \\ \text { 2. obese } & 557 \\ \text { 3. obesity } & 415 \\ \text { 4. type 2 diabetes } & 835 \\ \text { 5. NIDDM } & 234 \\ \text { 6. non insulin dependent diabetes } & 225 \\ \text { 7. non-insulin-dependent diabetes } & 214 \\ \text { 8. type II diabetes } & 143 \\ \text { 9. diet* } & 3171 \\ \text { 10. "weight loss" } & 608 \\ \text { 11. glycaemic control } & 138 \\ \text { 12. glycemic control } & 317\end{array}$

When using PubMed, it is necessary to combine searches using '\#' before the search number. The next stage is to combine the hits identified from different words that map to the same search term.

13. \#1 OR \#2 OR \#3

14. \#4 OR \#5 OR \#6 OR \#7 OR \#8

15. \#11 OR \#12

Now combine the hits identified from different search terms. 


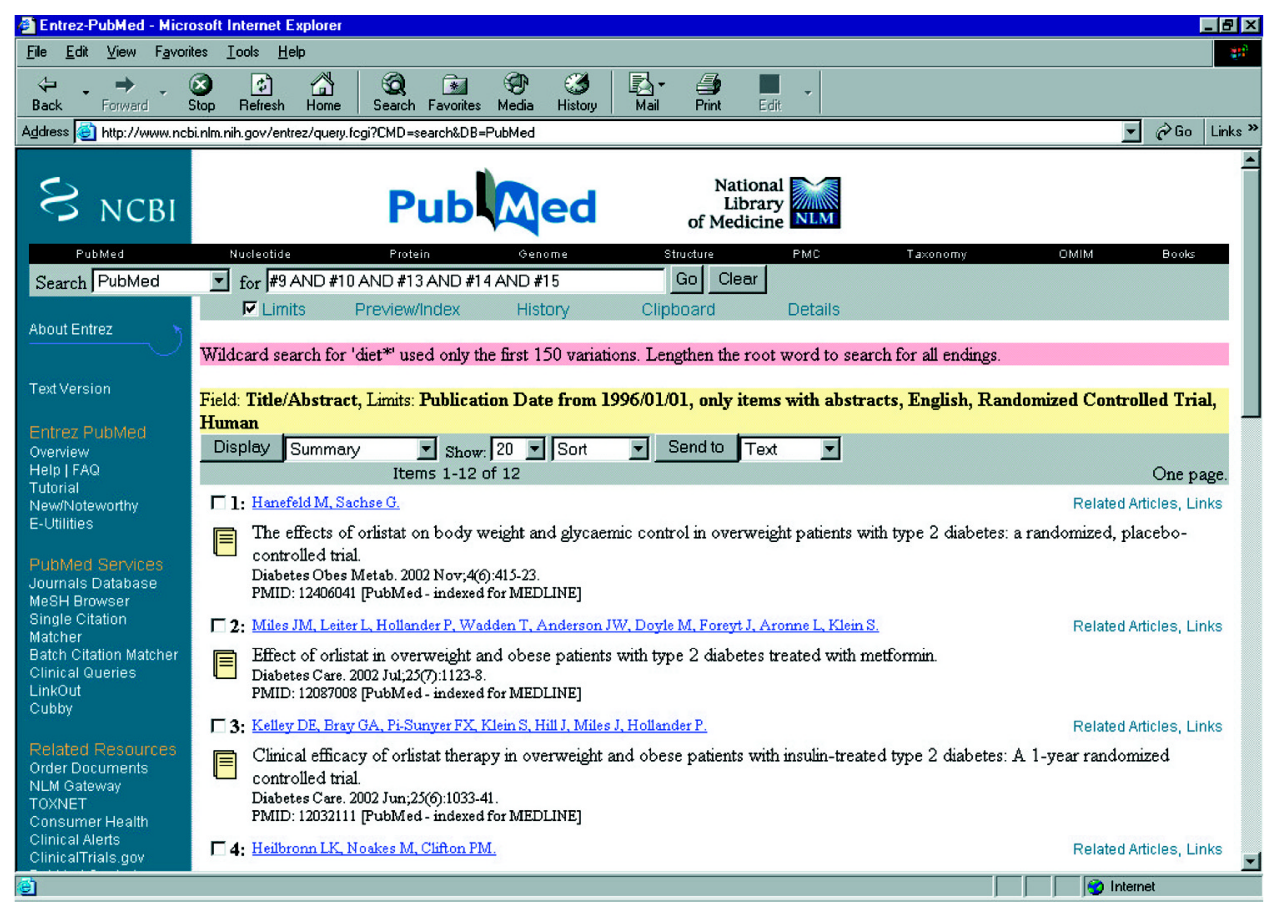

Fig. 2. Results of a search strategy in MEDLINE through PubMed. For details of search strategy, see p. 775.

\section{6. \#9 AND \#10 AND \#13 AND \#14 AND \#15}

This search returned twelve hits, as shown in Fig 2. By reading the titles of these hits, it will be obvious that some studies are probably irrelevant. Of the remainder, the aim should be to look through no more than ten 'hits'. If more than ten 'hits' are found, the search should be limited further. If no hits are found, the limits should be reduced. The results required should be in the abstract of the hits. If the full paper is needed, it may be available free on-line. If more than one study has been found, a decision should be made about which one is be used (not based on the results!).

\section{Smart searching in $10 \mathrm{~min}$}

If a few minutes are available to find the answer to the question, use Clinical Evidence. This book is also available on-line, via the National electronic Library for Health gateway.

For evidence on the effectiveness of interventions or initiatives for which randomised controlled trials have not been conducted (e.g. community or public health nutrition interventions), searching is more difficult. However, a number of websites are now developing useful databases of such interventions:
Health Development Agency Evidence Base: http:// www.hda-online.org.uk/html/research/evidencebase. html

National Institutes of Health: http://health.nih.gov/ Evidence for Policy and Practice Information and Co-ordinating Centre: http://eppi.ioe.ac.uk/EPPIWeb/ home.aspx

National Health Service Centre for Reviews and Dissemination: http://www.york.ac.uk/inst/crd/welcome.htm Effective Public Health Practice Project: http:// www.health.hamilton-went.on.ca/CSCARB/EPHPP/ ephpp.htm

Finally, a collaboration of academics from four UK universities (University College London, University of Oxford, University of Manchester and University of Teeside) is in the process of developing a database of best evidence of nutritional interventions, Systematic Reviews in Nutrition.

Systematic Reviews in Nutrition aims to facilitate making well-informed decisions about dietary recommendations in populations, and dietary interventions in health care and the community, by preparing, maintaining and promoting the accessibility of Systematic Reviews in Nutrition. It is hoped to have Systematic Reviews in Nutrition in working order by the end of 2003. 